\title{
Innovative approaches to support the global 2020 Round of Population and Housing Censuses
}

\author{
Oliver P. Fischer \\ U.S. Census Bureau, 4600 Silver Hill Road, Washington, DC 20746, USA \\ Tel.: +1 301763 6249; E-mail: Oliver.p.fischer@census.gov
}

\begin{abstract}
For over 65 years, the U.S. Census Bureau's International Programs (IP) has been providing capacity-strengtheningoriented technical assistance and training to National Statistical Offices around the world. This paper describes how IP has modernized and enhanced its public domain tools and technical assistance strategies to support low-and middle-income countries prepare for the global 2020 Round of Population and Housing Censuses.
\end{abstract}

Keywords: US Census Bureau, International Program, Population and Housing Census, capacity building, technical support

\section{Introduction}

Population and Housing Censuses provide a snapshot of the size and demographic, social, and economic makeup of a nation and each of its localities by counting each of its residents once - and only once - in their habitual place of residence. Census data serve as a basis for public resource allocation and for democratic discourse in the public domain, the media, and national decision-making bodies. The census enables policy makers to set informed targets on a variety of areas crucial for a society's well-being and development, including health, economic growth, poverty reduction, good governance, and democracy building. Moreover, the census is the pillar of a nation's statistical system and provides the sampling frame to improve the reliability of estimates derived from future surveys.

Realizing the benefits of a census requires the effective planning and implementation of one of the most massive and complex exercises a nation undertakes. The census involves everyone in the nation and, through the use of its output, serves everyone in the nation. The process entails delineating the entire country into enumeration areas, training and hiring a large number of field staff, canvassing all households and group living facilities, collecting information about each individual, transferring the data into electronic format, and analyzing and disseminating results - all within a brief and bounded timeframe [1]. Given the enormity and cost of this exercise, most nations conduct a census only once every ten years.

The responsibility for implementing the census often lies with the National Statistical Office (NSO). This responsibility is an opportunity for the NSO to highlight the importance of statistics and build the reputation of the office. Yet, in many developing countries, the resources required to conduct a census are often well beyond those available to the NSO. As such, the NSO and other host-country Ministries conduct a strategic donor outreach campaign, attempting to persuade international development partners to contribute resources to the census project. These resources are often utilized to pay the salaries of the large number of census field staff, to implement technological enhancements necessary to conduct a high quality census, and to develop the technical and managerial capacity of NSO staff. NSOs often seek to engage external partners to provide training and technical assistance. The U.S. Census Bureau is one of the leading providers of such assistance. 


\section{U.S. Census Bureau's International Programs: 65 years of sharing expertise}

The Census Bureau has a staff of nearly 40 experts dedicated to developing public domain tools and conducting technical assistance and on-the-job trainings to facilitate the process of implementing censuses in low and middle-income countries. This team, the International Programs (IP), consists of cartographers, statisticians, demographers, data processors, IT specialists, economists, and public health experts. IP is funded entirely by external partners, the largest and longest-standing of which is the United States Agency for International Development (USAID). This partnership has been in place for 65 years and has enabled the Census Bureau to share its expertise with nearly 80 countries, helping conduct over 130 censuses.

In preparation for the global 2020 Round of Population and Housing Censuses, IP has modernized and enhanced its public domain tools and developed its strategies to support statistical development in low- and middle-income countries at the global, regional, and county levels. These tools and strategies focus on supporting host-country government counterparts to become more self-reliant while producing high quality statistics that can build communities and strengthen democratic governance.

\section{Enhancing public domain tools to support the 2020 Round of censuses}

To determine the tools that could have the greatest impact for the 2020 Round of censuses, IP reflected on the current approaches for targeting and providing technical assistance to NSOs, considered the primary technological innovations since the 2010 Round of censuses, and assessed the utility of existing public domain tools. This review directed our focus towards three primary domains: enhancing technical and managerial needs assessments through improvements to the Tool for Assessing Statistical Capacity (TASC); modernizing our data capture and processing solution by implementing innovations to the Census and Survey Processing System (CSPro); and developing the Demographic Analysis and Population Projections System (DAPPS), a tool designed to facilitate the process of producing population estimates and projections. These tools and their application to the 2020 Round of censuses are detailed below.

Developing a work plan that effectively targets the primary needs of an NSO requires understanding the areas of relative strength and weakness across the institution. The TASC provides quantitative measures of an NSO's managerial and technical capacity to conduct a high-quality census or recurring household-based survey [2]. The results of the TASC assist NSOs, along with technical assistance providers such as IP, identify areas where additional training is needed and inform strategies for capacity-strengthening-oriented interventions. The TASC can be administered a second time following the implementation of the technical assistance program to measure the impact of the capacity-building efforts. In 2017, the Census Bureau updated the TASC to capture technological advances in mapping, data processing, and dissemination that NSOs in developing countries are considering as they prepare for their 2020 censuses. The TASC has been administered in over 60 countries and is widely adopted by governments and International Organizations around the world.

A fundamental prerequisite for producing quality and timely census results is the successful collection and processing of data. No matter how well census field staff have conducted enumeration, the quality of results will suffer if the data are improperly processed. CSPro, developed by the Census Bureau and initially released in 2000, is a public domain software package used by hundreds of organizations and tens of thousands of individuals for entering, editing, tabulating, and disseminating census and survey data [3]. The software is designed to be as user-friendly as possible, yet powerful enough to handle the most complex censuses and surveys.

Recent developments in technology and the subsequent decrease in the cost of electronic devices has made computer-assisted personal interviewing (CAPI) a viable and attractive option for census data-collection operations. In anticipation of this transition, the Census Bureau released CSPro Android in 2016 to provide NSOs a mobile data capture solution for their censuses and surveys. CSPro users, including NSO staff, can easily transfer the skills acquired over years of using CSPro to process data from traditional pencil-andpaper censuses to developing CSPro Android applications for CAPI censuses. Aside from the data collection application, CSPro features enable users to develop robust fieldwork monitoring systems, display interactive maps, and collect paradata to assess and improve data quality. The initial implementation of CSPro Android for a census was in Lesotho in 2016, which was the first mobile census conducted in Africa. Since then, low- and middle-income countries across the world have selected CSPro Android as the software 
solution of choice for their 2020 census with an estimated quarter of a billion people already enumerated by 2020 .

Following the processing of census data, NSOs must analyze and disseminate results suitable for a variety of data users. One area of technical support frequently requested by NSOs following a census is developing updated official population estimates and projections. Policymakers and planners around the world use population estimates and projections to forecast future demographic characteristics and plan for impending water, food, energy, and service demands. In preparation for the 2020 Round of censuses, the Census Bureau developed the Demographic Analysis and Population Projections System (DAPPS). DAPPS is designed to enable analysts from a variety of backgrounds to produce population estimates and projections with ease. The tool has a user-friendly spreadsheet interface and intuitive guides for its analysis and projections functions [4]. Demographers from over 70 countries have used DAPPS to produce estimates and projections.

\section{Leading global and regional initiatives to improve coordination and develop capacity}

The Census Bureau's leadership and support to developing country NSOs for the 2020 Round of censuses extends beyond development of tools. IP actively engages in multilateral partnerships to promote and facilitate the coordinatiozn of census support, conducts statistical development trainings at the global and regional levels, and is involved in numerous multi-year country-specific initiatives to prepare NSOs to conduct high-quality censuses.

In 2016, the Census Bureau, United Nations Population Fund (UNFPA), and the United Nations Statistics Division (UNSD) convened the International Committee on Census Coordination (ICCC) to enhance communication and coordination amongst the primary providers of census technical assistance to developing country NSOs. The objective of the ICCC is to promote the implementation of international best practices and coordinate planned, ongoing, and needed technical support for countries to implement successful censuses. This Committee meets multiple times per year to review regional and country-specific census preparations, highlight and share innovative approaches to building statistical capacity, and pursue avenues for improved collaboration. One recent success of the ICCC is the effort to reduce overall costs of im- plementing CAPI censuses for countries through the establishment of tablet sharing arrangements, which is currently being implemented in southern Africa. Since its formation, the ICCC has extended membership to the World Bank, improving census donors' awareness of some of the challenges NSOs face in implementing censuses. The ICCC has been instrumental in improving coordination amongst key census stakeholders, avoiding duplication of effort, and ensuring higherquality technical support across the world.

Guided by the belief that workshop participants benefit as much from each other as from instructors, the Census Bureau conducts multiple global and regional workshops per year. The Global Training Program, based at the Census Bureau's Headquarters near Washington, D.C., brings together NSO staff from every region of the world to participate in workshops focused on skill development in preparation for censuses. The workshops involve guest lecturers from across the Census Bureau who provide practical examples on census implementations in the United States and techniques for applying strategies in a variety of contexts. Since the beginning of the 2020 Round of censuses, over 150 individuals from 57 countries have participated in the Global Training Program for courses such as Census Planning and Management, CAPI for Subject Matter Specialists, and Introduction to Survey Sampling.

Our Regional Training Program addresses identified statistical needs in particular world regions through hands-on and applied workshops, often with the financial and logistical support of multiple international partners. Given our longstanding relationships with NSOs around the world and the adoption of the TASC in many countries, IP is able to gauge the primary statistical needs of world regions. Our Regional Training Program addresses these needs by bringing together NSOs at similar stages in the census lifecycle and providing targeted and context-appropriate training. Similar to the Global Training Program, the Regional Training Program gives participants the opportunity to learn from counterparts at multiple NSOs about approaches and potential solutions to census-taking challenges. Since the beginning of the 2020 Round of censuses, the Census Bureau conducted 16 regional workshops, training 454 participants from 76 countries.

\section{Supporting country NSOs with needs-tailored technical assistance and capacity building}

The most involved and in-depth technical assistance the Census Bureau provides is through our 
country-specific capacity strengthening initiatives. In this model, the Census Bureau and the host-country NSO utilize the TASC results to develop a work plan oriented towards building the skills of the NSO to conduct a high-quality census. Depending on the needs of the NSO and the funds available, these partnerships range from two to four years in duration and include between 5 and 35 short-term technical assistance visits by Census Bureau staff to the host country. The activities are structured as either on-the-jobtrainings or more formal classroom-style workshops. Generally, the technical areas requiring the most extensive assistance for our country-specific initiatives are census planning, pre-enumeration cartographic operations, and data processing.

One example of our country-specific work is our ongoing capacity-strengthening-oriented technical support to the National Statistical Office-Malawi (NSO) for their 2018 Population and Housing Census. At the request of the NSO and the USAID-Malawi Mission, Census Bureau staff traveled to Zomba, Malawi to administer the TASC. Over 70 NSO staff completed the TASC, the results of which highlighted training needs in the areas of cartography, data processing, data analysis, and the implementation of a CAPI census. Census Bureau staff, in collaboration with the NSO, developed a work plan comprising 29 short-term technical assistance missions to Malawi and two work-study visits to the United States to target the identified needs. A number of the trainings focused on strengthening the CSPro Android skills of the NSO data processing and subject matter staff to ensure successful implementation of a CAPI census. Malawi's enumeration took place over a two-week period in September 2018 and preliminary results were released within three months. This is record time for an African census and has been recognized as a major success by the international community. Our partnership with NSO-Malawi is currently focused on developing census thematic reports, utilizing DAPPS to produce population projections, and developing innovative data products.

\section{Conclusion: Innovating through self-reflection and a commitment to sustainable capacity building}

While we have been sharing our experience in censuses and surveys for over 65 years, the Census Bureau continues to assess and revise our approaches to providing the highest-quality technical assistance to our developing country NSO partners. Guided by the principals of self-reliance and improved decision-making, we are always searching for better ways to have a greater impact. Our recent innovations ahead of the 2020 Round of censuses have been the result of selfreflection and engagement with census stakeholders on the global, regional, and country levels. This search has led to our modernization of public domain tools, our leadership in global census coordination, our belief in peer-mediated capacity building at the global and regional levels, and our deep technical engagement with NSO counterparts.

\section{References}

[1] United Nations. Principles and Recommendations for Population and Housing Censuses, Revision 3; 2015.

[2] U.S. Census Bureau. Tool for Assessing Statistical Capacity. August 2017 [cited 20198 Aug]. Available from: https:// www.census.gov/data/software/tasc.html.

[3] U.S. Census Bureau. Census and Survey Processing System. April 2019 [cited 20198 Aug]. Available from: https://www. census.gov/data/software/cspro.html.

[4] U.S. Census Bureau. Demographic Analysis and Population Projections. April 2019 [cited 20198 August]. Available from: https://www.census.gov/data/software/dapps.html. 